\title{
Murine cutaneous responses to the rocky mountain spotted fever vector, Dermacentor andersoni, feeding
}

\section{Dar M. Heinze ${ }^{1}$, J. Russ Carmical ${ }^{1,2}$, Judith F. Aronson ${ }^{1}$, Franscisco Alarcon-Chaidez ${ }^{1}$, Stephen Wikel ${ }^{3}$ and Saravanan Thangamani ${ }^{1,4,5}$ *}

${ }^{1}$ Department of Pathology, University of Texas Medical Branch, Galveston, TX, USA

${ }^{2}$ Department of Biochemistry and Molecular Biology, University of Texas Medical Branch, Galveston, TX, USA

${ }^{3}$ Department of Medical Sciences, Quinnipiac University, Hamden, CT, USA

${ }^{4}$ Institute for Human Infections and Immunity, University of Texas Medical Branch, Galveston, TX, USA

${ }^{5}$ Galveston National Laboratory, University of Texas Medical Branch, Galveston, TX, USA

\section{Edited by:}

Sukanya Narasimhan, Yale

University School of Medicine, USA

Reviewed by:

Juan Anguita, CIC bioGUNE, Spain

Jesus G. Valenzuela, National

Institute of Allergy and Infectious

Diseases, USA

\section{*Correspondence:}

Saravanan Thangamani, Galveston National Laboratory, Department of Pathology, Institute for Human

Infections and Immunity, University of Texas Medical Branch, Galveston, TX-77555, USA

e-mail: sathanga@utmb.edu
Tick salivary glands produce complex cocktails of bioactive molecules that facilitate blood feeding and pathogen transmission by modulating host hemostasis, pain/itch responses, wound healing, and both innate and adaptive immunity. In this study, cutaneous responses at Dermacentor andersoni bite-sites were analyzed using Affymetrix mouse genome arrays and histopathology at 12, 48, 96 and $120 \mathrm{~h}$ post- infestation (hpi) during primary infestations and $120 \mathrm{hpi}$ during secondary infestations. The microarray data suggests: (1) chemotaxis of neutrophils, monocytes, and other cell types; (2) production and scavenging of reactive oxygen species; and, (3) keratin- based wound healing responses. Histological analysis supported the microarray findings. At $12 \mathrm{hpi}$, a mild inflammatory infiltrate was present in the dermis, especially concentrated at the junction between dermal connective tissue and underlying adipose tissue. A small lesion was located immediately under the hypostome and likely represents the feeding "pool." Surprisingly, at $48 \mathrm{hpi}$, the number of inflammatory cells had not increased from $12 \mathrm{hpi}$, perhaps mirroring the reduction in gene expression seen at this time point. The feeding lesion is very well defined, and extravasated erythrocytes are readily evident around the hypostome. By $96 \mathrm{hpi}$, the inflammatory infiltrate has increased dramatically and the feeding lesion appears to have moved deeper into the dermis. At $120 \mathrm{hpi}$, most of the changes at $96 \mathrm{hpi}$ are intensified. The infiltrate is very dense, the epidermis is markedly thickened, the feeding lesion is poorly defined and the dermal tissue near the hypostome appears to be loosing its normal architecture. In conclusion, during $D$. andersoni feeding infiltration of inflammatory cells increases across time concurrent with significant changes in the epidermal and dermal compartments near the feeding tick. The importance of changes in the epidermal layer in the host response to ticks is not known, however, it is possible the host attempts to "slough off" the tick by greatly increasing epithelial cell replication.

Keywords: tick, tick feeding, tick saliva, Immunomodulation, Dermacentor andersonii

\section{INTRODUCTION}

Hard (Ixodid) ticks are important vectors of disease world- wide, making them a significant threat to public health. The process of tick feeding is an extended interplay between the tick, tick salivary molecules, and the host response. Tick saliva is a complex mixture of molecules that has been shown to inhibit a broad range of host responses and facilitate pathogen transmission, while host responses can, in some cases, inhibit the feeding process and block pathogen transmission (Titus et al., 2006; Kazimírová and Štibrániová, 2013; Wikel, 2013) Hard ticks have been divided into two main phylogenetic lineages, the prostriates (containing the genus Ixodes) and the metastriates (containing all other hard tick genera). The divergence between these lineages is estimated to have occurred 124 million years ago (range from 101 to 166) (Mans et al., 2012). While salivary proteins from prostriate and metastriate lineages differ in amino acid composition, they accomplish similar goals in terms of inhibiting host responses to allow successful blood feeding. However, some differences are likely based on differential vectorial capacity. In this study, cutaneous host responses to $D$. andersoni were measured in an effort to understand host factors effecting pathogen transmission. Affymetrix GeneChip Mouse Genome 430A 2.0 arrays were used to measure host gene expression in the skin of $D$. andersoni infested mice at $12,48,96$, and $120 \mathrm{~h}$ post- infestation (hpi) during primary infestations and $120 \mathrm{hpi}$ during secondary infestations. In addition, histopathological analysis of bite-site lesions from primary infestation time points and quantitative real-time PCR analysis of lymph nodes from secondary infestation time points were analyzed. These studies allow us to describe the cutaneous host response during primary and secondary infestations, measure changes in gene expression patterns across time, view potential patterns related to tick immuno-suppression, correlate 
the histopathology analysis to the gene expression data, and measure systemic responses in the draining lymph node. These analyses are important for understanding the context of pathogen transmission and tick rejection at the tick-host inGAterface.

\section{METHODS \\ TICKS}

Pathogen-free D. andersoni colonies were maintained in our laboratory as described (Bouchard and Wikel, 2005; Heinze et al., 2012a,b). All life cycle stages were kept in sterile glass vials with mesh tops in desiccators at $22^{\circ} \mathrm{C}$ containing saturated solutions of $\mathrm{KNO}_{3}$ to obtain desired relative humidity with a $16: 8 \mathrm{~h}$ photoperiod. For routine colony maintenance adult and/or nymphal ticks were fed on New Zealand white rabbits and nymphs and/or larvae were fed on mice.

\section{ANIMALS}

BALB/c mice used in this study were obtained from The Jackson Laboratory (Bar Harbor, ME). Mice were cared for in accordance with a protocol approved by the Institutional Animal Care and Use Committee (IACUC) of the University of Texas Medical Branch.

\section{TIME COURSE INFESTATIONS}

To perform time course infestations, $6-8$ week old female BALB/c mice were placed into individual restrainers or anesthetized with a $150 \mu \mathrm{l}$ intraperitoneal injection containing $10 \mathrm{mg} / \mathrm{mL}$ ketamine (Fort Dodge Animal Health, Fort Dodge, IA) and $1 \mathrm{mg} / \mathrm{mL}$ xylazine (Phoenix Pharmaceutical, St. Joseph, MO) in sterile PBS (Gibco, Life Technologies, Carlsbad, CA) and infested with pathogen-free nymphal ticks. Ticks were allowed to attach for approximately $1 \mathrm{~h}$ and unattached ticks were discarded. Mice were then removed from restraints and housed individually. Secondary infestations involved two rounds of infestation. Mice were infested with nymphal ticks that were allowed to complete their feeding cycle (4-5 days). 14 days after the last primary infestation tick completed feeding, mice were re-infested with nymphal ticks using the same protocol described above. Bite sites were analyzed at $12,48,96$, and 120 hpi during primary infestations, and at 120 hpi during secondary infestations. Three mice were measured at each time point in all micro-array or PCR-array experiments; controls consisted of 3 similarly housed but tick-free mice. Skin biopsies ( $4 \mathrm{~mm}$ ) at the tick feeding loci (around ear) were harvested and stored in RNAlater (Ambion) at $-20^{\circ} \mathrm{C}$ for RNA extraction or fixed in $10 \%$ neutral buffered formalin for $48 \mathrm{~h}$ and then stored in $70 \%$ ethanol at $4^{\circ} \mathrm{C}$ for histopathology. For histopathology, skin biopsies were harvested with ticks attached.

\section{RNA ISOLATION}

Ticks were removed from all skin biopsies before RNA extraction. Tissue samples were homogenized individually in $1 \mathrm{~mL}$ Trizol (Life Technologies, Carlsbad, CA) using an Ultra-Turrax T8 (Ika, Wilmington, NC) tissue disperser. The Trizol protocol was followed until phase separation. The aqueous phase was retained and one volume $70 \%$ ethanol (Acros Organics) was added and the samples applied to RNeasy micro kit (Qiagen) columns. The RNeasy protocol was then followed, including the in-column
DNase digestion step. One extra wash step with buffer RW1 and two extra wash steps with buffer RPE facilitated the isolation of high quality RNA. All samples were eluted in nuclease-free water. After extraction, RNA was quantitated spectrophotometrically using a NanoDrop ND-1000 (NanoDrop Technologies, $\mathrm{DE})$. All samples were required to read greater than 1.8 on both $A_{260} / A_{280}$ and $A_{260} / A_{230}$ ratios. For subsequent microarray analysis, quality of the purified RNA was assessed by visualization of 18 and $28 \mathrm{~S}$ RNA bands using an Agilent BioAnalyzer 2100 (Agilent Technologies, CA). Resulting electropherograms were used in the calculation of the $28.18 \mathrm{~S}$ ratio and the RNA Integrity Number, which was greater than 6.8 in all samples (Schroeder et al., 2006). For subsequent real-time PCR analysis, RNA integrity was determined by denaturing (formaldehyde) agarose gel electrophoresis followed by staining with Sybr Gold stain (Invitrogen). Visualization of clear ribosomal bands indicated minimal degradation. Eluted RNA samples were aliquoted and stored at $-80^{\circ} \mathrm{C}$ until used.

\section{HOST GENE EXPRESSION PROFILING USING AFFYMETRIX GENECHIPS}

Total RNA (500 ng) was converted to cRNA for microarray analysis using the Ambion MessageAmp ${ }^{\mathrm{TM}}$ Premier RNA Amplification Kit (Life Technologies Corporation, CA) according to manufacturer's instructions. Total fragmented cRNA (10 vg) was hybridized to the Affymetrix GeneChip Mouse Genome 430A 2.0 array according to the manufacturer's (Affymetrix, CA) conditions. The chips were washed and stained in a GeneChip Fluidics Station 450 and fluorescence detected with an Affymetrix-7G Gene Array scanner using the Affymetrix GeneChip Command Console software (AGCC1.1). The resulting CEL files were uploaded to iReport ${ }^{\mathrm{TM}}$ (Ingenuity Systems), and the default data analysis was utilized. The resulting values were then filtered for $p$-values $\leq 0.05$ and a fold change $\leq-1.5$ or $\geq+1.5$ and these data are reported as significant findings (Figure 1).

\section{GENE ONTOLOGY(GO) ANALYSIS}

Broad patterns of significantly modulated genes were visualized using heatmaps plotted in the $\mathrm{R}$ package gplots (Warnes et al., 2013). Two gene lists were used to generate these maps; the first included all genes modulated during the primary infestation and the second included all genes modulated at any time point in the study (Figure 2). These heat maps suggested modulated genes grouped into early primary infestation (12-96 hpi), late primary infestation ( $120 \mathrm{hpi})$, and secondary infestation patterns $(120 \mathrm{hpi})$. The significantly modulated genes in these groups were compiled and used to generate a Venn diagram (Figure 3) through an online interactive tool (Oliveros, 2007). The lists of unique and shared genes in each group identified in the Venn diagram were analyzed using the Database for Annotation, Visualization, and Integrated Discovery (DAVID) (Huang da et al., 2009a,b) website using the Affymetrix GeneChip Mouse Genome 430A 2.0 array as a background. The functional annotation clustering tool was used to group similar gene ontology terms into clusters with a mathematically-generated ranking score. These clusters can then be named based on the gene ontology terms in each cluster, giving a concise over-view of the results (Table 1). 


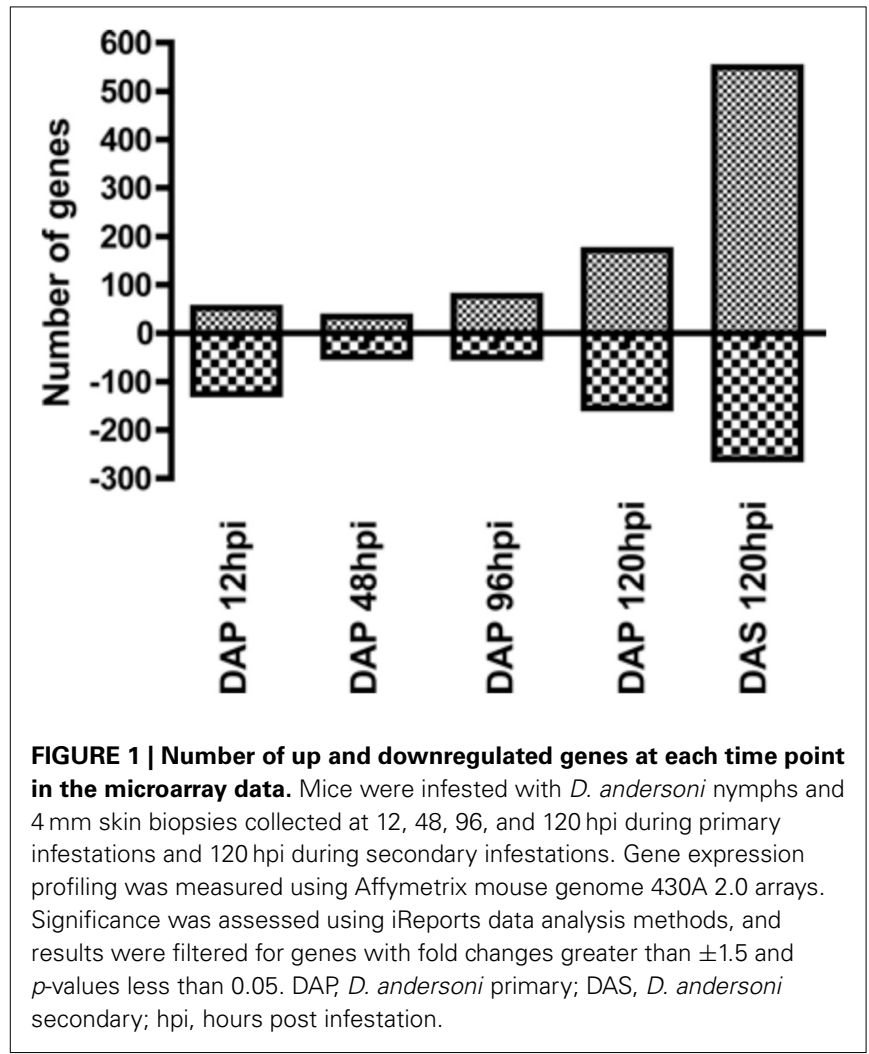

\section{VALIDATION OF ARRAY DATA}

The GeneChip results were validated by an additional experiment. A separate set of mice were infested with ticks and tissues harvested as described above. For the D. andersoni microarray experiment, gene targets (Table 2 ) were validated at 12,48 , 96 , and $120 \mathrm{hpi}$ during primary infestations and $120 \mathrm{hpi}$ during secondary infestations using 3 mice/time point. Primers were purchased from Integrated DNA Technologies; these primer sequences are provided in Table 3. Primers were mixed with $\mathrm{RT}^{2}$ SYBR green qPCR master mix (Qiagen) and aliquoted into iCycler iQ PCR plates (Bio-Rad) using an epMotion 5075 automated pipetting system (Eppendorf). Plates were sealed and stored at $-20^{\circ} \mathrm{C}$ until use. For each real-time PCR run, the $\mathrm{RT}^{2}$ First Strand Kit (Qiagen) was used to convert $1 \mathrm{vg}$ total RNA into cDNA, which was then loaded onto PCR plates using the epMotion 5075 automated pipetting system (Eppendorf). In some cases, the epMotion system was not functioning properly and the plates were loaded with primer and/or cDNA using an 8channel pipette. These plates were run on an iCycler iQ5 real-time PCR instrument (Bio-Rad) with the following cycling protocol: $10 \mathrm{~min}$ at $95^{\circ} \mathrm{C} ; 15 \mathrm{~s}$ at $95^{\circ} \mathrm{C}, 1 \mathrm{~min} 60^{\circ} \mathrm{C}$ for 40 cycles, and an 80 -cycle $\left(+0.5^{\circ} \mathrm{C} /\right.$ cycle $) 55-95^{\circ} \mathrm{C}$ melt curve. Every run included hypoxanthine guanine phosphoribosyl transferase (Hprt) and heat shock protein 90 alpha (Hsp90ab1) as endogenous control genes, and "no template" and "no first strand" controls. HTqPCR, an R-based program for real-time PCR data analysis (Dvinge and Bertone, 2009), was used to analyze data using the delta-delta Ct method for gene expression normalization and measurement, and the linear models in microarray analysis (LIMMA) package for statistical comparisons between infested and tick-free mice.

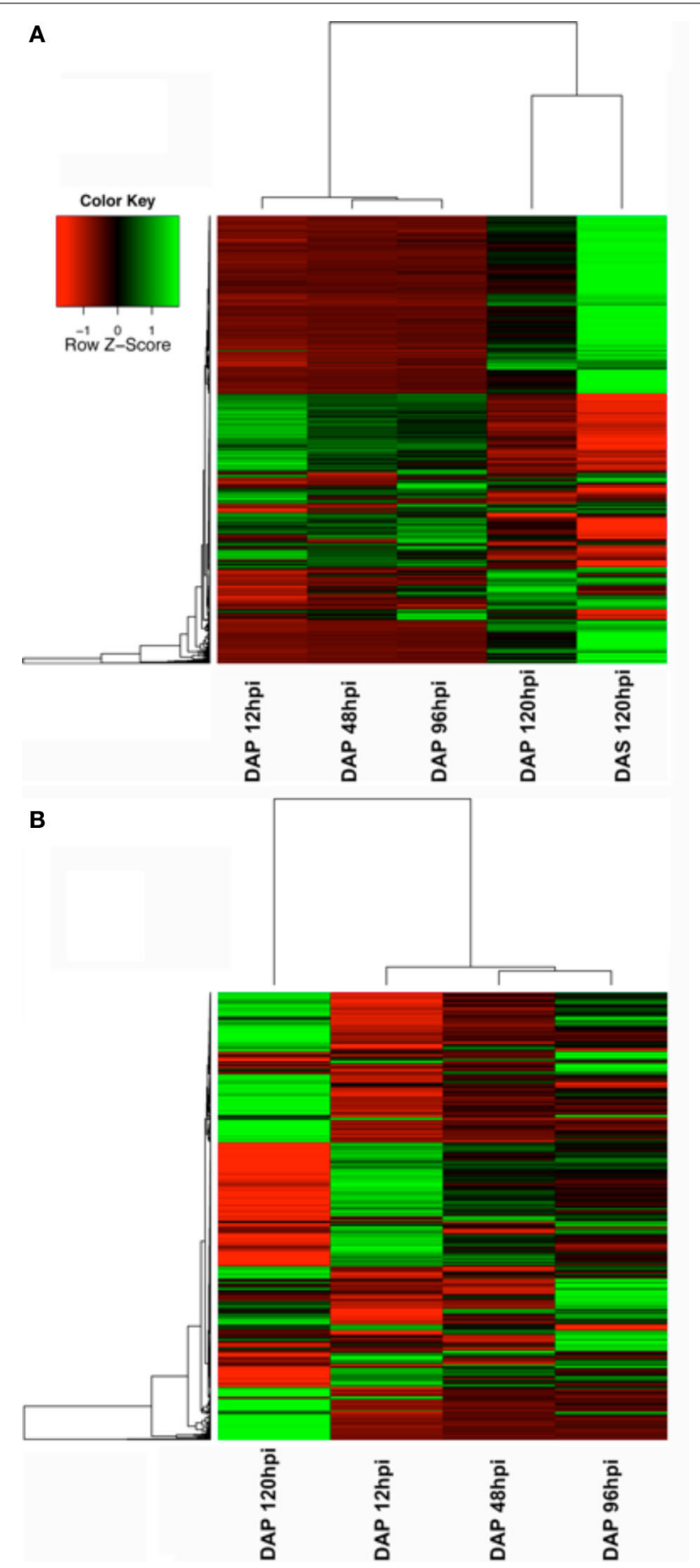

FIGURE 2 | Heatmaps showing changes in gene expression across time. (A) Changes in genes significant at any time point in the microarray study. (B) Changes in genes significant during the primary infestation only.

\section{QUANTITATIVE REAL-TIME PCR ANALYSIS OF MOUSE LYMPH NODES AFTER D. ANDERSONI FEEDING}

Draining cervical/auricular lymph nodes were harvested from mice used for validation of the $D$. andersoni microarray data set during secondary infestations at $120 \mathrm{hpi}$. Quantitative real-time PCR analysis was undertaken using the gene targets in Table 4. 


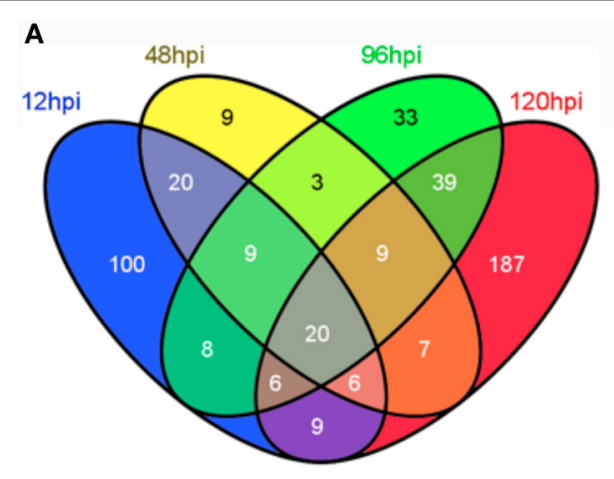

FIGURE 3 | Venn diagram showing the overlap of significantly modulated genes between time points in the microarray study. (A) Venn diagram for primary infestation time points. (B) Venn diagram for early time points (DAP12, 48, and $96 \mathrm{hpi}$ ), later time
B

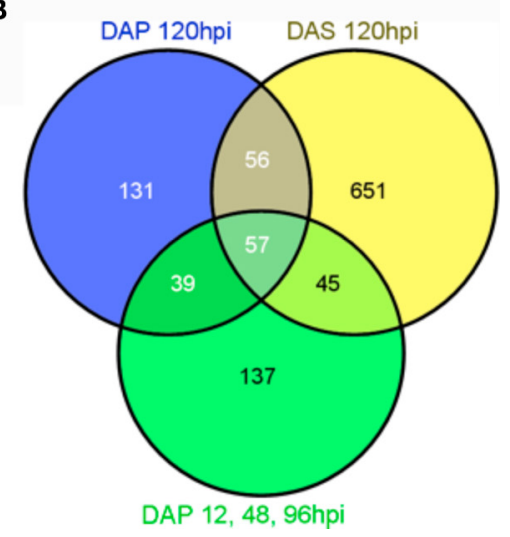

points (DAP120 hpi), and secondary infestation (DAS120 hpi). The Venn diagram in B was constructed based on variations in gene expression profiles suggested in the heatmaps. All Venn diagrams were created using (Oliveros, 2007).
These results were compared against lymph nodes from tick-free mice. RNA was isolated and PCR run as described above.

\section{RESULTS AND DISCUSSION}

The number of up and downregulated genes meeting our filtering criteria at each time point is shown in Figure 1. Although there was a small decrease in the number of upregulated genes between 12 and $48 \mathrm{hpi}$, the general trend was an increasing number of upregulated genes across time. The downregulated genes show a different profile, with more genes modulated at early and late time points than those in the middle. A list of all genes modulated at any time point (Supplement data 1) in the study was used generate a heatmap (Figure 2A). This heatmap suggested a similar gene expression profile for the first three time points (12, 48 , and 96 hpi during primary infestations) that then shifted to a second pattern that was similar between 120 hpi in primary and secondary infestations. This suggested there was a shift in gene expression profiles between early and late time points, as well as between primary and secondary infestations.

To provide a higher-resolution analysis of the primary infestation time points, a second heatmap was constructed using only the significantly modulated genes in the primary infestation (Figure 2B). While this heatmap shows some differences between 12,48 , and $96 \mathrm{hpi}$, the overall similarity seen in the first heatmap was preserved. In addition, the dramatic change in expression profile between the first three time points and the 120 hpi primary infestation time point was maintained.

A Venn diagram for the primary infestation time points (Figure 3A) shows more uniquely modulated genes at $12 \mathrm{hpi}$ and 120 hpi than at other time points, again suggesting differences between early and late responses. To explore the patterns observed in the heatmaps, all significantly modulated genes from 12, 48, and 96 hpi primary infestation were combined into a single data set. This list, the list of modulated genes at 120 hpi primary infestation, and the list of modulated genes at 120 hpi secondary infestation were used to create a Venn diagram (Figure 3B). This revealed 137 genes unique to early primary infestations, 131 genes unique to late primary infestations, 651 genes unique to late secondary infestations, and 57 genes shared across all time points. These lists, along with the lists of genes shared between each group were submitted to the Database for Annotation, Visualization, and Integrated Discovery (DAVID) bioinformatics database using the Affymetrix Mouse Genome 430A 2.0 array as a background list. The functional annotation-clustering tool was used to group similar ontology terms together, and the top ten clusters containing significant ontology terms $(p<0.05)$ were named based on the terms included (Table 1).

\section{THE CORE RESPONSE (GENES SHARED BETWEEN ALL TIME POINTS)}

Analyzing the shared response between all time points allows the description of a "core" response to tick feeding. Genes in the core response were significant at early, primary, late primary, and late secondary. Significant gene ontology clusters were chemotaxis and inflammation, cation homeostasis, intermediate filaments and keratinization, carbohydrate binding, and regulation of oxidatives stress.

Genes in the chemotaxis and inflammation clusters were chemokines, cytokines, and anti-microbial molecules. Chemokines Ccr1, Ccl2, Ccl6, Ccl7, Ccl12, Cxcl1, Cxcl2, and Pf4 (platelet factor 4 or $\mathrm{Cxcl} 4$ ) were upregulated and are consistent with monocyte and neutrophil migration into the bite site. Additional chemoattractants for neutrophils such as the S100 molecules (calgranulins) and Saa3 (serum amyloid A3) were also upregulated. Antimicrobial proteins such as Lcn2 (lipocalin 2) and Ptx3 (pentraxin related gene) that function in iron sequestration and opsonization respectively were upregulated. These results combined with the upregulation of IL-1b suggest a potent innate-like immune response dominated by macrophages and neutrophils is a hallmark of the anti-tick response.

Gene ontology terms in clusters 3 and 6 were related to cations or metal ions. Genes in these clusters related to ion regulation were $\mathrm{Mt1}$ and $\mathrm{Mt} 2$ (metallothionein); these molecules are involved in regulating copper and zinc, but also are involved in reactive oxygen species scavenging, cell transcription, and 
Table 1 | Top ten clusters from DAVID analysis based on lists from Figure 3B Venn diagram.

\begin{tabular}{|c|c|c|}
\hline \multicolumn{2}{|c|}{ Core } & DAP12-48-96 only \\
\hline 1 & $\begin{array}{l}\text { Chemotaxis, immune } \\
\text { response, response to } \\
\text { wounding }\end{array}$ & $\begin{array}{l}1 \text { Response to DNA damage and } \\
\text { cellular stress, DNA repair }\end{array}$ \\
\hline 2 & Chemotaxis, cell migration & $\begin{array}{l}2 \text { Intracellular } \\
\text { non-membrane-bounded } \\
\text { organelle }\end{array}$ \\
\hline 3 & Cation homeostasis & 3 Nucleotide and ATP binding \\
\hline 4 & $\begin{array}{l}\text { Intermediate filament, } \\
\text { cytoskeleton organization and } \\
\text { structure }\end{array}$ & $\begin{array}{l}4 \text { Regulation of transcription, DNA } \\
\text { binding }\end{array}$ \\
\hline 5 & $\begin{array}{l}\text { Keratinization, keratinocyte } \\
\text { and epidermal cell } \\
\text { differentiation }\end{array}$ & 5 Circadian rhythm \\
\hline 6 & Response to metal ion & $\begin{array}{l}6 \text { Chromosome, chromatin binding } \\
\text { and organization }\end{array}$ \\
\hline 7 & Carbohydrate binding & 7 Nuclear body, nucleoplasm \\
\hline 8 & Regulation of blood pressure & 8 mRNA metabolic process \\
\hline 9 & & 9 Regulation of transcription \\
\hline 10 & & 10 Cardiac muscle contraction \\
\hline & P120-DAP12-48-96 & DAP120 only \\
\hline 1 & $\begin{array}{l}\text { No clusters with significant } \\
\text { terms }\end{array}$ & $\begin{array}{l}1 \text { Regulation of cell and } \\
\text { cell-substrate adhesion }\end{array}$ \\
\hline 2 & & 2 Blood vessel development \\
\hline 3 & & 3 Embryonic development \\
\hline 4 & & $\begin{array}{l}4 \text { Nuclear or intracellular organelle } \\
\text { lumen }\end{array}$ \\
\hline 5 & & 5 RNA processing \\
\hline 6 & & 6 Cell growth \\
\hline 7 & & 7 MAP kinase activity \\
\hline 8 & & $\begin{array}{l}8 \text { Positive regulation of transcription } \\
\text { and RNA metabolic process }\end{array}$ \\
\hline 9 & & 9 Meisis (cell cycle) \\
\hline DA & P120-DAS120 & DAS120 \\
\hline 1 & Extracellular region & $\begin{array}{l}1 \text { Inflammatory and defense } \\
\text { response }\end{array}$ \\
\hline 2 & $\begin{array}{l}\text { Proteinaceous extracellular } \\
\text { matrix }\end{array}$ & $\begin{array}{l}2 \text { Leukocyte and lymphocyte } \\
\text { activation, T cell differentiation }\end{array}$ \\
\hline 3 & Cell surface & $\begin{array}{l}3 \text { Positive regulation of immune } \\
\text { response, immune } \\
\text { response-regulating cell surface } \\
\text { receptor signaling pathway }\end{array}$ \\
\hline 4 & Response to wounding & 4 Lysosome \\
\hline 5 & Cellular homeostasis & $\begin{array}{l}5 \text { T cell activation, leukocyte and } \\
\text { lymphocyte proliferation }\end{array}$ \\
\hline 6 & Fatty acid biosynthetic process & 6 Chemotaxis \\
\hline 7 & $\begin{array}{l}\text { Regulation of phosphorylation, } \\
\text { protein kinase activity }\end{array}$ & 7 Regulation of cytokine production \\
\hline 8 & & 8 Regulation of immune cells \\
\hline 9 & & $\begin{array}{l}9 \text { Regulation of adaptive immune } \\
\text { response }\end{array}$ \\
\hline 10 & & 10 Endocytosis \\
\hline
\end{tabular}

(Continued)
Table 1 | Continued

\section{DAS120-DAP12-48-96}

1 Keratinocyte, epidermal cell

differentiation

2 Rhythmic process

The core analysis represents significantly modulated genes shared between early primary (DAP12, 48, 96), late primary (DAP120), and secondary infestations (DAS120). DAP, D. andersoni primary; DAS, D. andersoni secondary.

Table 2 | Gene list for quantitative real-time PCR validation of the $D$. andersoni microarray study.

\begin{tabular}{llll}
\hline Arg1 & Cxcl5 & il4 & S100a9 \\
Ccl12 & & Il6 & sele \\
Ccl6 & Hprt & Irak4 & Serpine1 \\
Ccl7 & Hsp90ab1 & krt16 & Socs3 \\
Ccr1 & ifng & krt6b & Stat3 \\
Chi3l1 & il10 & Map3k6 & Wnk1 \\
Clec10a & il12a & Mt1 & No RT \\
Clec4e & II1b & ptges & No temp \\
\hline
\end{tabular}

immune responses. Studies with metallothionein knockout mice suggest Mt1 and 2 play protective roles in inflammatory settings (Inoue et al., 2009; Thirumoorthy et al., 2011). Xanthine dehydrogenase, an enzyme involved in the oxidative metabolism of purines, was also upregulated. Xanthine dehydrogenase may be converted to xanthine oxidase by sulfhydryl oxidation, and xanthine oxidase can be an important source of reactive oxygen species (Sakuma et al., 2012). Thus the generation and scavenging of reactive oxygen species appears to be the primary function of the genes in these clusters.

Clusters 4 and 5 were dominated by gene ontology terms related to intermediate filaments and keratinization. Indeed, keratin intermediate filaments Krt6a, 6b, and 16 were upregulated. Krt16 has been shown to pair with either Krt6a or $6 \mathrm{~b}$ to form essential intermediate filaments in epithelial wound repair. Specifically, these intermediate filaments are required to support keratinocyte migration into the wound site (Paladini et al., 1996; Wong and Coulombe, 2003). In addition to these molecules, Sprr1b (small proline rich $1 \mathrm{~b}$ ) was upregulated. The encoded protein is a significant component of the cornified cell envelope that maintains a permeability barrier across epithelial tissues (Patel et al., 2003). Downregulated genes included a minor collagen (type 11 alpha 1), the cytoskeletal component spectrin, and a little-studied molecule Limch1 (LIM and calponin homology domains 1). These results suggest a keratin intermediate filamentbased wound healing response is activated at the tick feeding lesion, however it is surprising that other cytoskeletal elements are downregulated, a pattern that is even more striking in the early primary infestation as discussed below. Thus keratin intermediate filaments but not other cytoskeletal molecules are activated in response to tick bites.

Gene ontology cluster 7 was related to carbohydrate binding. The primary molecules in this group were c-type lectin receptors Clec4d and Clec4n, although other molecules such as 
Table 3 | List of gene targets and primer sequences used in the $D$. andersoni quantitative real-time PCR validation of the microarray data and also in quantitative real-time PCR analysis of lymph nodes at $\mathbf{1 2 0} \mathrm{hpi}$ during secondary infestations.

\begin{tabular}{|c|c|c|}
\hline Gene name & Primer $1\left(5^{\prime}-3^{\prime}\right)$ & Primer $2\left(5^{\prime}-3^{\prime}\right)$ \\
\hline RORgt & Ccgctgagagggcttcac & Tgcaggagtaggccacattac \\
\hline Gapdh & gtggagtcatactggaacatgtag & Aatggtgaaggtcggtgtg \\
\hline $\mathrm{Tb} \times 21$ & caagaccacatccacaaacatc & Ttcaaccagcaccagacag \\
\hline Arg1 & Agtgttgatgtcagtgtgagc & Gaatggaagagtcagtgtggt \\
\hline Stat4 & Caactcctctgtcaccatgt & Cgtcaaagctatgtccagtga \\
\hline Chi3l1 & Ccatcaaagccataagaacgc & ccagaaacaccaacctgaaga \\
\hline $\mathrm{Hdc}$ & Gaccgaatcacaaaccacag & tctacctccgacatgccaa \\
\hline Irak4 & Cagcagtagttgaggttcacg & Acacccaaatctgacatctaca \\
\hline Stat1 & gacttcagacacagaaatcaactc & Ttgacaaagaccacgcctt \\
\hline Stat6 & agttcttcctgcttccgatg & Gccaccatcagacaaatacttc \\
\hline Tgfb1 & cgtggagtttgttatctttgctg & Gacgtcactggagttgtacg \\
\hline Stat3 & Agctcctcagtcacgatca & gttcaagcacctgacccttag \\
\hline Ptges & Caggaatgagtacacgaagcc & Gtattacaggagtgacccagatg \\
\hline$\| 12 a$ & ctctcgttcttgtgtagttcca & acagatgacatggtgaagacg \\
\hline Foxp3 & Ctgtcttccaagtctcgtctg & Ctggtctctgcaggtttagtg \\
\hline Lcn2 & Cctgtgcatatttcccagagt & Ctacaatgtcacctccatcctg \\
\hline$\| 1 b$ & Cgagatttgaagctggatgc & Tgacagtgatgagaatgacctg \\
\hline$\| 17 a$ & gagcttcccagatcacagag & Agactacctcaaccgttcca \\
\hline$\| 10$ & Atggccttgtagacaccttg & Gtcatcgatttctcccctgtg \\
\hline Sele & Cctgattgttttgaacctagacg & Cgtcctcattgctctacttgt \\
\hline Stat5a & Cgcttgattctttcagtgaca & Tgagaacacccgcaatgag \\
\hline Ccl6 & Gaagtgtcttgaaagccttgatg & Agaaactccaagactgccat \\
\hline Hprt & Aacaaagtctggcctgtatcc & Ttccctggttaagcagtacag \\
\hline Serpine1 & ggctgagatgacaaaggct & Tcacaagtctttccgaccaag \\
\hline 116 & Gcaagtgcatcatcgttgttc & Agtcggaggcttaattacacat \\
\hline Gata3 & gtccccattagcgttcctc & Ccttatcaagcccaagcgaa \\
\hline Ifng & Agataatctggctctgcagga & Gtcattgaaagcctagaaagtctg \\
\hline Hsp90ab1 & Cctgaaaggcaaaggtctcc & Ccaccctgctctgtactact \\
\hline Clec10a & gaccaaggagagtgctagaag & tgactgagttcctgcctct \\
\hline Wnk1 & Aaaggcatggttcaaaaggtc & Gcagatctaccgtcgagtga \\
\hline Cor1 & Aggaactggtcaggaataatagc & Caaaggcccagaaacaaagtc \\
\hline$\| 4$ & Tgatgctctttaggctttccag & Cagagactctttcgggcttt \\
\hline Clec4e & Cttatggtggcacagtcctc & Agtggcaatgggtggatg \\
\hline S100a9 & ccatcagcatcatacactcctc & Tggaagcacagttggcaa \\
\hline Map3k6 & Gatttccggggccatatactg & Cacagagacatcaagggagac \\
\hline Krt6b & Ctgcttttgtacgcttgttga & Gacagcatcattggagagagg \\
\hline $\mathrm{Ccl} 7$ & Tttgtttcttgacatagcagcat & Tctcactctctttctccacca \\
\hline Cxcl5 & Gatccagacagacctccttct & ttgatcgctaatttggaggtga \\
\hline Mt1 & gctcttcttgcaggaggtg & Tcacttactccgtagctccag \\
\hline Krt16 & cagctcattctcgtacttggtc & Tcaaagactacagcccctact \\
\hline Actb & Cgatggaggggaatacagc & Tctttgcagctccttcgtt \\
\hline Socs3 & ggaaacttgctgtgggtga & Gagatttcgcttcgggacta \\
\hline Ccl12 & Ggaggcatagaagtgtggaaa & agagacactggttcctgact \\
\hline
\end{tabular}

Ccl7, Pf4, and Ptx3 were also present. C-type lectins are expressed on dendritic cells and macrophages, and play important roles in orchestrating the inflammatory response. Their importance host responses to tick feeding is strongly suggested by studies showing the I. scapularis salivary protein Salp 15 can bind and activate DC-sign (CD209) (Hovius et al., 2008). Clec4n (dectin-2) is
Table 4 | Gene list for quantitative real-time PCR analysis of mouse lymph nodes after secondary infestation with $D$. andersoni for $120 \mathrm{~h}$.

\begin{tabular}{lll}
\hline Actb & $\| 12 a$ & Stat3 \\
Foxp3 & $\| 17 a$ & Stat4 \\
Gapdh & $\| 1 b$ & Stat5a \\
Gata3 & $\| 4$ & Stat6 \\
Hprt & $\| 6$ & Tbx21 \\
Hsp90ab1 & RORgt & Tgfb1 \\
Ifng & Socs3 & No RT \\
I10 & Stat1 & No temp \\
\hline
\end{tabular}

thought the associate with $\mathrm{FcR} \gamma$ and signal through a Syk-Card9 pathway to activate NFkB (Geijtenbeek and Gringhuis, 2009). Ligands include mannose, fungal antigens, and dust mites (Patel et al., 2003), although others likely exist. The precise role of ctype lectins in tick feeding is unknown, but these results suggest they may act as important pattern recognition receptors and may modulate downstream responses.

The final cluster contained terms related to regulation of blood pressure. This primary gene in this group, Wnk1 (lysine deficient protein kinase 1), is downregulated and it influences blood pressure by regulating salt absorption in the kidney. Wnk1 also plays a similar role in sweat glands (Kahle et al., 2008). Upregulated genes in this group include Hmox1 (heme oxygenase 1), and Gch1 (GTP cyclohydrolase 1). Hmoxl can play a role in protecting tissues from oxidative damage, while Gch1 can produce either NO or reactive oxygen species depending cofactor availability. Thus the gene ontology terminology may be misleading here, as the genes appear more related to regulating oxidative status than blood pressure.

\section{EARLY PRIMARY INFESTATION}

Gene ontology analysis of the early primary infestation revealed a number of clusters related to nucleotide processing such as DNA repair, nucleotide binding, transcription, and mRNA metabolism. A review of the genes represented by these clusters suggested they could be broadly grouped into DNA repair molecules, DNA helicases of the SWI/SNF family, transcription factors, components of the spliceosome, and mRNA metabolism. DNA helicases of the SWI/SNF family are not active helicases, but function as DNA translocases thought to modulate chromatin structure (Durr et al., 2006). Thus most of these molecules can be related to transcription, from chromatin remodeling, transcription factor binding, RNA splicing, and mRNA metabolism. Interestingly, nearly all of these genes were downregulated, suggesting that transcription is decreased early in the primary infestation. Of the few upregulated transcriptional regulators, Nfkbia and Tsc22d3 were of particular interest because they have been shown to inhibit NFkB and AP-1 pro-inflammatory pathways (Beaulieu and Morand, 2011). DNA repair molecules were also downregulated. In contrast, molecules in the circadian rhythm cluster, such as Per1 were upregulated. Overexpression of Per1 in tumor cells was shown to increase DNA-damage induced apoptosis and significantly decreased cell growth in untreated cells (Gery et al., 2006). This suggests some connection between DNA repair, circadian rhythm molecules, and growth arrest in the skin at the 
bite site may exist. Terms in the final cluster were related to cardiac muscle contraction, although review of these genes and genes in the highly significant (though un-clustered) GO term actin binding (GO:0003779) revealed molecules related to muscle contraction, actin binding, and cytoskeletal adaptor molecules that may relate signaling events to changes in the cytoskeleton. Cytoskeletal elements have been shown to play vital roles in epithelial wound healing processes by aiding cell migration and wound contracture (Abreu-Blanco et al., 2012). Thus the early host responses were characterized by a reduction in transcription, DNA repair, inhibition of inflammation, potential inhibition of the cell cycle, and downregulation of cytoskeletal elements. These effects suggest that tick feeding may inhibit early aspects of the wound healing response.

When the genes shared between early and late primary infestation were submitted to DAVID, no significant clusters were observed.

\section{LATE PRIMARY INFESTATION}

The first three clusters (regulation of adhesion, blood vessel development, embryonic development) and clusters 6 and 7 (cell growth, MAPK activity) share similar genes and are treated together here. In the process of normal epidermal wound healing, repair must take place in the epidermis, dermis, and vasculature to restore normal function. Particularly for the re-epithelialization of the wound, keratinocytes must loose some of their adhesiveness and migrate into the wound area. This process shares similar characteristics to the epithelial-mesenchymal transition (EMT) (Nakamura and Tokura, 2011) during embryonic development. In support of a similar process at the tick bite site, genes known to be involved in EMT such as Tdgf1 (teratocarcinoma-derived growth factor 1 or Cripto-1), Cyr61 (cysteine rich protein 61 or CCN1), and Smad5 (SMAD family member 5 , a TGF- $\beta$ signaling intermediate) were upregulated. While not specific to EMT, the MAPK signaling pathway is known to be important (Leopold et al., 2012), increasing the relevance of cluster 7. Additional upregulated genes that may be involved were Tnfrsf12s (tumor necrosis factor receptor superfamily, member 12a), Junb (Jun-B oncogene), and Epgn (epithelial mitogen). While a specific role for the encoded proteins in EMT has not been delineated, these molecules may be related to TNF- $\alpha$, AP-1, and growth factor responses that are known to be involved (Leopold et al., 2012).

Downregulated molecules included extracellular matrix components Colla1 (collagen type I alpha 1) and Lamb2 (laminin beta 2), calcium ion channel or signaling molecules Tesc (tescalcin) and $\mathrm{Pkd} 2$ (polycyctic kidney disease 2), integrin ligand Edil3 (EGF-like repeats and discoiding I-like domains 3), and growth factor related molecules Pdgfrb (platelet derived growth factor receptor beta) and Tgfb3 (transforming growth factor beta 3 ). In the final analysis, a convincing case for EMT in wound healing responses during late primary infestations cannot be made. However, changes in adhesion, cell growth, MAPK activity, and the appearance of genes normally associated with embryonic development such as Tdgfl are suggestive of a related process.

The remaining clusters were nuclear or organelle lumen, RNA processing, positive regulation of transcription, and meiosis or cell cycle related. The majority of the genes in these clusters code for RNA binding proteins, RNA processing proteins, and transcriptional activators/transcription factors. An interesting theme among the RNA-related molecules was an interaction with rRNA, implying some interaction with ribosomes. Most of the genes in these clusters were upregulated, contrasting with the transcription-related genes early in the primary infestation. Thus, the data suggests transcription is inhibited during early host responses, but then activated near the end of the feeding cycle.

Genes shared between 120 hpi primary and 120 hpi secondary infestation clustered into a number of broad categories such as extracellular region, cell surface, cellular homeostasis, and regulation of phosphorylation. Two clusters were more specific, however. Fatty acid biosynthesis contained three genes, Fcerla (IgE Fc receptor), Scd3 (stearoyl-coenzyme A desaturase), and Ch25h (cholesterol 25-hydroxylase). Fcer1a and Ch25h were upregulated, while Scd3 was downregulated. Fcerla encodes the alpha chain of the FceR, a receptor that binds IgE with high affinity. This receptor is activated by antigen-induced cross-linking, and induces many downstream responses including the release of histamine and the production of prostaglandins D2 and E2 (Ugajin et al., 2011). Supporting this role, genes encoding proteins involved in prostaglandin E synthesis are upregulated at 120 hpi primary infestation, although they are not shared with 120 hpi secondary infestation.

Ch25h encodes a protein that catalyzes the formation of 25hydroxycholesterol (Lund et al., 1998), a molecule that can act as a chemoattractant for migrating cells but also inhibits the function of B cells (Diczfalusy et al., 2009) and can promote the survival of Listeria monocytogenes infected cells (Zou et al., 2011). Scd3 is an important enzyme in the synthesis of monounsaturated fatty acids, many of which are subsequently used as components of membrane phospholipids. Interestingly, decreasing fatty acid synthesis inhibited rift valley fever virus replication in mouse embryonic fibroblasts (Moser et al., 2012), suggesting fatty acid synthesis may aid viral replication. These genes suggest a role for fatty acid metabolites and the control of fatty acid synthesis at the tick bite site. Finally, the response to wounding cluster contained pro-inflammatory genes such as Il-6, Cxcl5, and P2ry12 (purinergic receptor $\mathrm{P} 2 \mathrm{Y}$ ) and extracellular matrix molecules Cd44 and Timp3 (tissue inhibitor of metalloproteinase 3). These genes are involved in inflammation and cell migration, although they are often associated with acute inflammation rather than appearing late in the induction of the immune response as they do here.

In summary, late in the primary infestation the innate immune response remains the major player. Keratin-based wound healing responses and the suggestion of an EMT-type transition driving wound re-epithelialization are present. In addition, transcription appears to be potentiated in contrast to earlier in the primary infestation. Genes shared with the secondary infestation suggest fatty acid metabolism and the response to wounding are important in host responses near the end of the feeding cycle.

\section{SECONDARY INFESTATION}

There were 651 genes modulated only in the secondary infestation. Gene ontology analysis of this group of genes resulted in many clusters related to the immune response. These clusters shared many genes with each other and they will be discussed in 
relation to what appeared to be the most distinctive feature(s) of each cluster or group of clusters.

The first cluster contained GO terms related to inflammatory and defense responses. Genes in this group supported four primary processes: complement (synthesis), coagulation (pathway synthesis), Toll-like receptor/IL-1 response, and acute phase response. Clusters 2, 5, 8, and 9 were leukocyte and lymphocyte activation, $\mathrm{T}$ cell activation and leukocyte proliferation, regulation of immune cells, and regulation of adaptive immune response, respectively. These clusters shared similar genes, and supported the importance of protein kinases (SRC family, Syk, and Jak), T-cell costimulation (CD40, CD86, CD3d, and Lck), cytokines, immunoreceptors (cytokine, antibody, C-type lectin, and Toll-like receptors 1 and 4), and signaling intermediates in immunoreceptor signaling. Cluster 3, positive regulation of immune response, contained many similar features. However, two genes strongly related to iron handling in the innate immune response, Slc1lal (solute carrier family 11 member 1) and Hpx (hemopexin) were upregulated. Cluster 4 contained GO terms related to lysosomes. The largest group of upregulated molecules in this cluster were genes encoding many different cathepsins. Cathepsins are lysosomal proteinases that function primarily in protein turnover in the lysosome (Kuester et al., 2008). However, additional roles for cathepsins include (but are not limited to) antigen presentation (Yamamoto et al., 2012), keratinocyte differentiation (Yamamoto et al., 2012), itch responses (Andoh et al., 2012), and wound healing (Akira and Takeda, 2004). The chemotaxis cluster contained chemokines $\mathrm{Ccl} 24, \mathrm{Ccl} 17, \mathrm{Ccl} 5, \mathrm{Ccl} 4, \mathrm{Ccl} 8$, $\mathrm{Ccl} 3$, and $\mathrm{Cxcl} 16$ consistent with the migration of lymphocytes, monocytes, neutrophils, eosinophils, and basophils into the bite site. Additional molecules that supported cell migration such as integrins, chemoattractants, and intracellular molecules that regulate changes in cell shape were also upregulated. Cluster 7 contained terms related to regulation of cytokine production. These genes code for proteins that function as immunoreceptors, cytokine signaling intermediates, and cytokines. Finally, the endocytosis cluster contained genes encoding classical endocytotic proteins such as Clta (clathrin, light polypeptide) and picalm (phosphatidylinositol binding clathrin assembly protein). Other genes in this cluster were antibody Fc receptors Fcerlg, Fcgr3, and Fcgr2b and lectin recepors Clec7a, CD209a CD209d, CD209e, and $\mathrm{Mrcl}$. These receptors may function in endocytosis by binding antibodies or carbohydrate moieties and triggering endocytotic machinery. This may be an important method of delivering tick-derived antigens to the endosomal/lysosomal compartment where they could be processed for antigen presentation.

These results suggest the importance of complement, coagulation, innate immune responses, and adaptive immunity in the host response to $D$. andersoni nymphs. In particular, the importance of Toll-like receptors 1 and 4 and lectin pattern recognition receptors appear to be important aspects of the innate immune response during secondary infestations. Both TLRs and C-type lectins have been shown to be important pattern recognition receptors for sensing pathogens and initiating and shaping innate and adaptive immune responses (Akira and Takeda, 2004; Geijtenbeek and Gringhuis, 2009). Both TLR2 and DC-SIGN (CD209e) have already been shown to be important in anti-tick responses by modulating cytokine responses (Hovius et al., 2008; Oliveira et al., 2010). These studies support our results and also suggest the importance of these receptors is likely broader than previously reported. Within the adaptive immune response, these results suggest the capture, processing, and presentation of antigens is an important process during secondary infestations. In particular, cathepsins may be important proteins in this process with possible contributions to wound healing and/or itch responses. The array results also support the co-stimulation and activation of lymphocytes.

\section{VALIDATION}

A list of genes was chosen to validate the microarray data (Table 2) using quantitative real-time PCR. Genes were chosen based on significant fold change in the microarray study that were involved in important pathways identified in the gene ontology analysis. Trends of up- and downregulation were well preserved between the microarray and validation study (Figure 4). In general, more genes had significant fold changes in the microarray that the validation for 12 and $48 \mathrm{hpi}$, and the validation had more significant fold changes from $96 \mathrm{hpi}$ onwards. At the $120 \mathrm{hpi} \mathrm{sec-}$ ondary infestation time point, all the gene targets validated except Chi3l1 and Wnk1. It should be noted that some genes significant only at later time points in the array study became significant earlier in the validation study (i.e., Arg1, Cxcl5), while some genes significant early in the array study did not become significant until later time points in the validation (i.e., Ccl12, Ccl7, Krt6b, Krt16, and S100a9). Interestingly, the trend of more significant gene modulation at 12 than 48 hpi seen in the array data was upheld in the validation study.

In summary, the validation study upheld the microarray study with a few minor differences. Samples used for the validation study were derived from a completely separate infestation experiment than the array study, making it an extremely rigorous test and likely contributing to the small variations seen between data sets.

\section{LYMPH NODE STUDY}

The host response to ticks has frequently been reported to be polarized toward the production of Th2 cytokines. However, in our earlier work with I. scapularis, the gene expression profile in the skin was consistent with a more mixed response (Heinze et al., 2012a,b). Consistent with these results, gene expression profiling in the skin at $D$. andersoni bites did not yield a conclusive picture of CD4+ T cell polarization. While IL- 4 was present, so were IL-6 and IL-1b, cytokines more associated with Th17 responses than Th2. To assay the systemic host response, the expression of a panel of genes (Table 4) related to CD4+ T cell differentiation was measured in lymph node samples from naïve and 120 hpi secondary infestation mice. Important transcription factors for T regulatory cells (Foxp3), Th17 cells (Rorgt), Th1 cells (Tbx21) and Th1 cytokine (IL-12a) were either downregulated or unchanged between infested and control mice. In contrast, the Th2 cytokine IL-4 was upregulated (Figure 5). Thus, the results of this experiment strongly suggest the systemic response to repeated $D$. andersoni nymphal feeding in mice is polarized toward a Th2 profile. 


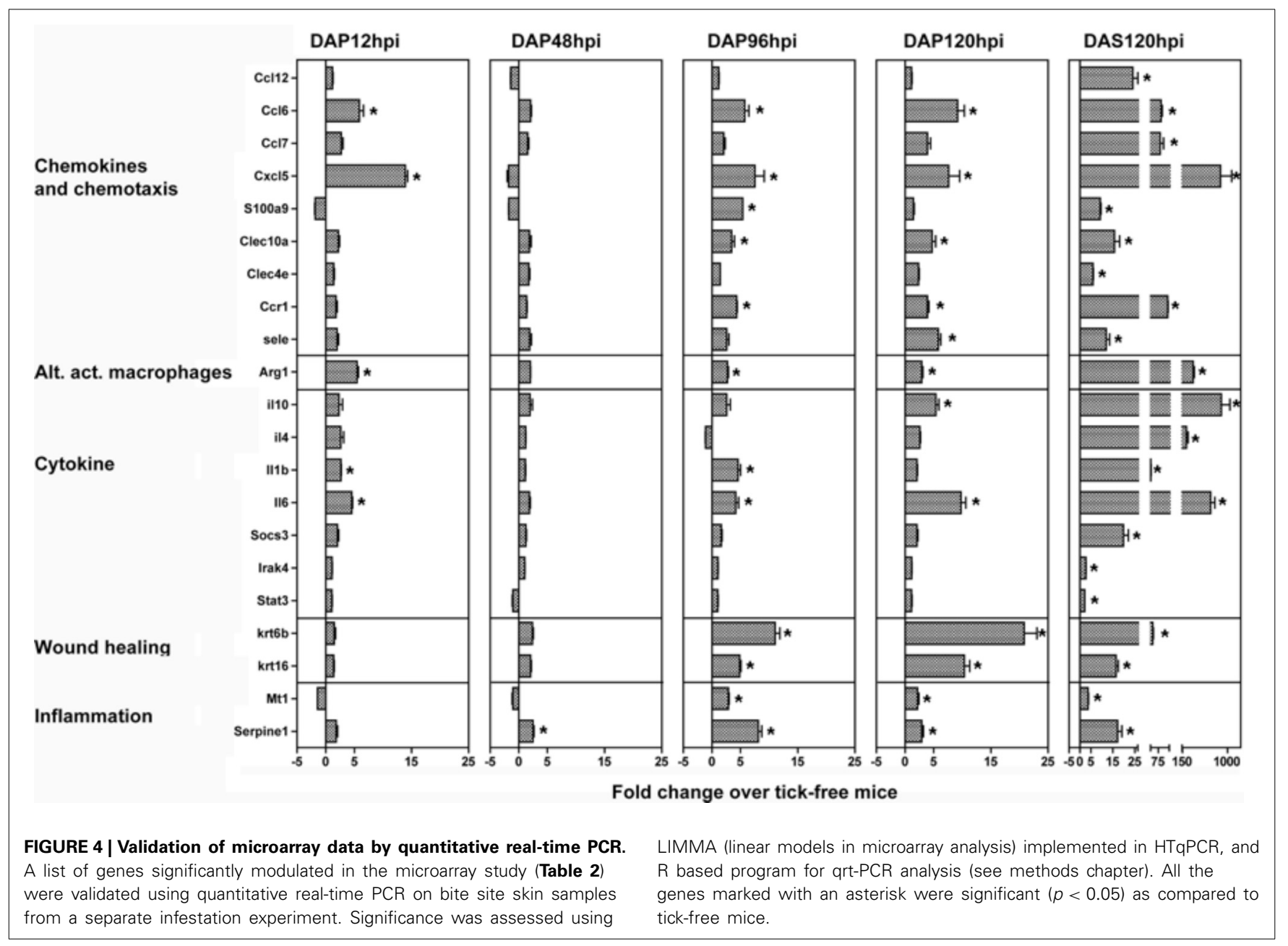

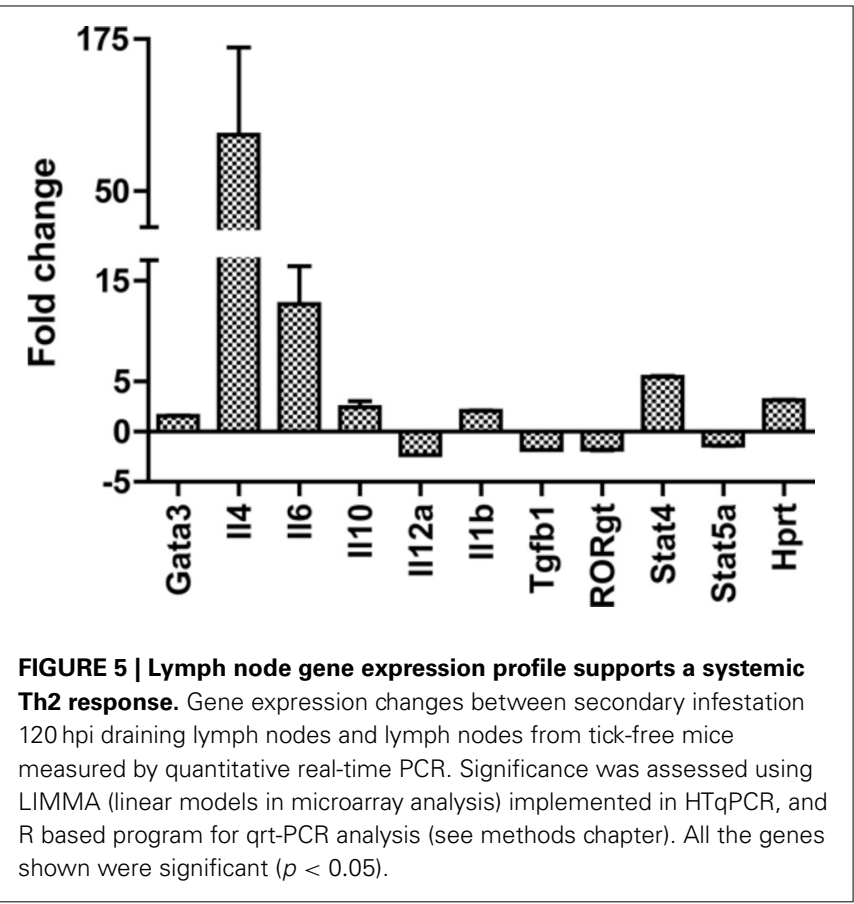

\section{HISTOLOGY}

The bite site is characterized by a large quantity of attachment cement secreted by the tick to anchor it to the host (Figure 6). The amount of cement increases throughout the feeding period, and it contains readily evident layers, suggesting that additional cement is secreted in waves throughout the feeding cycle. The deepest penetration of the hypostome into the skin is very shallow, in most cases barely passing through the epidermis. At $12 \mathrm{hpi}$, a mild inflammatory infiltrate was present in the dermis, especially concentrated at the junction between dermal connective tissue and underlying adipose tissue. A small lesion was located immediately under the hypostome and likely represents the feeding "pool." At $48 \mathrm{hpi}$, the number of inflammatory cells had not increased from $12 \mathrm{hpi}$, mirroring the reduction in gene expression seen at this time point. The feeding lesion is very well defined, and extravasated erythrocytes are readily evident around the hypostome. Some thickening of the epidermis is evident. By $96 \mathrm{hpi}$, the inflammatory infiltrate has increased dramatically and the feeding lesion appears to have moved deeper into the dermis. Epidermal thickening and extravasated erythrocytes and leukocytes are present. At $120 \mathrm{hpi}$, most of the changes at $96 \mathrm{hpi}$ are intensified. The infiltrate is very dense, the epidermis is markedly thickened, the feeding lesion is poorly defined and the dermal 


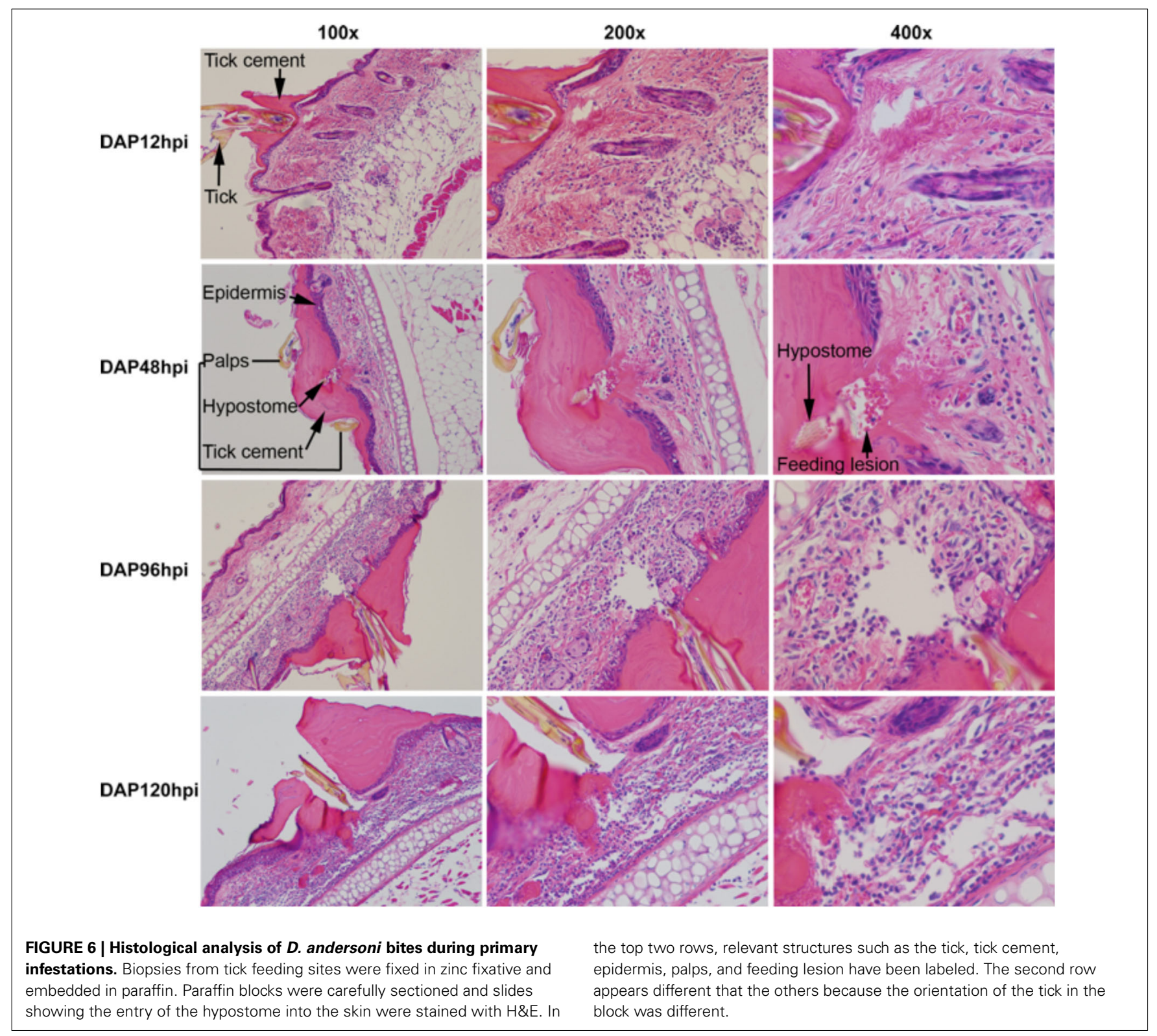

tissue near the hypostome appears to be loosing its normal architecture. Extravasated erythrocytes and leukocytes are prominent. In the microarray, the chemotaxis of immune cells into the bite site, and aspects of wound healing responses were especially prominent. The histological analysis supports these basic conclusions, showing an inflammatory infiltrate that increases across time, and significant changes in the epidermal and dermal compartments near the feeding tick.

\section{CONCLUSIONS}

The microarray data suggests the chemotaxis of neutrophils, monocytes, and other cell types, the production and scavenging of reactive oxygen species, keratin-based wound healing responses, and C-type lectins are important host responses to tick feeding conserved across primary and secondary infestations. Early time points in the primary infestation imply the inhibition of transcription and RNA processing. This inhibitory profile may represent tick-induced modulation of early host responses. Later time points in the primary infestation provide additional support for wound healing activities including the suggestion of EMT. The secondary infestation is characterized by increases in these same pathways, along with the addition of complement, coagulation, Toll-like receptors, acute phase response, antigen presentation, and the activation of lymphocytes. These results were supported by quantitative real-time PCR validation. The systemic murine response to $D$. andersoni nymphal feeding was polarized toward a Th2, based on real-time PCR analysis of draining lymph nodes from secondary infestations. The histological analysis supported the chemotaxis of neutrophils and monocytes into the bite site and the marked thickening of the epithelial layers may be the result of the upregulation of genes related to keratinization and wound healing. In conclusion, during $D$. andersoni 
feeding infiltration of inflammatory cells increases across time concurrent with significant changes in the epidermal and dermal compartments near the feeding tick. The importance of changes in the epidermal layer in the host response to ticks is not known, however, it is possible the host attempts to "slough off" the tick by greatly increasing epithelial cell replication.

\section{ACKNOWLEDGEMENTS}

This work is supported by the departmental start-up funds provided to Saravanan Thangamani, by NIAID/NIH \# UC7AI0 94660. We would like to thank Nicole Hauser for the help with tick rearing. Dar M. Heinze was supported NIAID/NIH \# T32AI007526.

\section{SUPPLEMENTARY MATERIAL}

The Supplementary Material for this article can be found online at: http://www.frontiersin.org/journal/10.3389/fmicb.2014.

\section{8/abstract}

\section{REFERENCES}

Abreu-Blanco, M. T., Watts, J. J., Verboon, J. M., and Parkhurst, S. M. (2012). Cytoskeleton responses in wound repair. Cell. Mol. Life Sci. 69, 2469-2483. doi: 10.1007/s00018-012-0928-2

Akira, S., and Takeda, K. (2004). Toll-like receptor signalling. Nat. Rev. Immunol. 4, 499-511. doi: 10.1038/nri1391

Andoh, T., Yoshida, T., Lee, J. B., and Kuraishi, Y. (2012). Cathepsin E induces itch-related response through the production of endothelin-1 in mice. Eur. J. Pharmacol. 686, 16-21. doi: 10.1016/j.ejphar.2012.04.024

Barrett, N. A., Maekawa, A., Rahman, O. M., Austen, K. F., and Kanaoka, Y. (2009). Dectin-2 recognition of house dust mite triggers cysteinyl leukotriene generation by dendritic cells. J. Immunol. 182, 1119-1128. doi: 10.4049/jimmunol.182.2.1119

Beaulieu, E., and Morand, E. F. (2011). Role of GILZ in immune regulation, glucocorticoid actions and rheumatoid arthritis. Nat. Rev. Rheumatol. 7, 340-348. doi: 10.1038/nrrheum.2011.59

Bouchard, K. R., and Wikel, S. K. (2005). "Care, maintenance, and experimental infestation of ticks in the laboratory setting," in Biology of Disease Vectors, 2nd Edn., ed W. C. Marquart (San Diego, CA: Elsevier Academic Press), 705-711.

Diczfalusy, U., Olofsson, K. E., Carlsson, A. M., Gong, M., Golenbock, D. T., Rooyackers, O., et al. (2009). Marked upregulation of cholesterol 25hydroxylase expression by lipopolysaccharide. J. Lipid Res. 50, 2258-2264. doi: 10.1194/jlr.M900107-JLR200

Durr, H., Flaus, A., Owen-Hughes, T., and Hopfner, K. P. (2006). Snf2 family ATPases and DExx box helicases: differences and unifying concepts from high-resolution crystal structures. Nucleic Acids Res. 34, 4160-4167. doi: 10.1093/nar/gkl540

Dvinge, H., and Bertone, P. (2009). HTqPCR: high-throughput analysis and visualization of quantitative real-time PCR data in R. Bioinformatics 25 3325-3326. doi: 10.1093/bioinformatics/btp578

Geijtenbeek, T. B., and Gringhuis, S. I. (2009). Signalling through C-type lectin receptors: shaping immune responses. Nat. Rev. Immunol. 9, 465-479. doi: $10.1038 /$ nri2569

Gery, S., Komatsu, N., Baldjyan, L., Yu, A., Koo, D., and Koeffler, H. P. (2006). The circadian gene perl plays an important role in cell growth and DNA damage control in human cancer cells. Mol. Cell 22, 375-382. doi: 10.1016/j.molcel.2006.03.038

Heinze, D. M., Carmical, J. R., Aronson. J. F., and Thangamani, S. (2012a). Early immunologic events at the tick-host interface. PLOS ONE 7:e47301. doi: 10.1371/journal.pone.0047301

Heinze, D. M., Wikel, S. K., Thangamani, S., and Alarcon-Chaidez, F. J. (2012b). Transcriptional profiling of the murine cutaneous response during initial and subsequent infestations with Ixodes scapularis nymphs. Parasit. Vectors 5:26. doi: 10.1186/1756-3305-5-26

Huang da, W., Sherman, B. T., and Lempicki, R. A. (2009a). Bioinformatics enrichment tools: paths toward the comprehensive functional analysis of large gene lists. Nucleic Acids Res. 37, 1-13. doi: 10.1093/nar/ gkn923

Huang da, W., Sherman, B. T., and Lempicki, R. A. (2009b). Systematic and integrative analysis of large gene lists using DAVID bioinformatics resources. Nat. Protoc. 4, 44-57. doi: 10.1038/nprot.2008.211

Hovius, J. W., de Jong, M. A., den Dunnen, J., Litjens, M., Fikrig, E., van der Poll, T., et al. (2008). Salp15 binding to DC-SIGN inhibits cytokine expression by impairing both nucleosome remodeling and mRNA stabilization. PLoS Pathog. 4:e31. doi: 10.1371/journal.ppat.0040031

Inoue, K., Takano, H., Shimada, A., and Satoh, M. (2009). Metallothionein as an anti-inflammatory mediator. Mediators Inflamm. 2009:101659. doi: $10.1155 / 2009 / 101659$

Kahle, K. T., Ring, A. M., and Lifton, R. P. (2008). Molecular physiology of the WNK kinases. Annu. Rev. Physiol. 70, 329-355. doi: 10.1146/annurev.physiol. 70.113006.100651

Kazimírová, M., and Štibrániová, I. (2013). Tick salivary compounds: their role in modulation of host defences and pathogen transmission. Front. Cell. Infect. Microbiol. 20:43 doi: 10.3389/fcimb.2013.00043

Kuester, D., Lippert, H., Roessner, A., and Krueger, S. (2008). The cathepsin family and their role in colorectal cancer. Pathol. Res. Pract. 204, 491-500. doi: 10.1016/j.prp.2008.04.010

Leopold, P. L., Vincent, J., and Wang, H. (2012). A comparison of epithelialto-mesenchymal transition and re-epithelialization. Semin. Cancer Biol. 22, 471-483. doi: 10.1016/j.semcancer.2012.07.003

Lund, E. G., Kerr, T. A., Sakai, J., Li, W. P., and Russell, D. W. (1998). cDNA cloning of mouse and human cholesterol 25-hydroxylases, polytopic membrane proteins that synthesize a potent oxysterol regulator of lipid metabolism. J. Biol. Chem. 273, 34316-34327. doi: 10.1074/jbc.273.51.34316

Mans, B. J., de Klerk, D., Pienaar, R., de Castro, M. H., and Latif, A. A. (2012). The mitochondrial genomes of nuttalliella namaqua (Ixodoidea: Nuttalliellidae) and argas africolumbae (Ixodoidae: Argasidae): estimation of divergence dates for the major tick lineages and reconstruction of ancestral blood-feeding characters. PLoS ONE 7:e49461. doi: 10.1371/journal.pone.0049461

Moser, T. S., Schieffer, D., and Cherry, S. (2012). AMP-activated kinase restricts rift valley fever virus infection by inhibiting fatty acid synthesis. PLoS Pathog. 8:e1002661. doi: 10.1371/journal.ppat.1002661

Nakamura, M., and Tokura, Y. (2011). Epithelial-mesenchymal transition in the skin. J. Dermatol. Sci. 61, 7-13. doi: 10.1016/j.jdermsci.2010.11.015

Oliveira, C. J., Carvalho, W. A., Garcia, G. R., Gutierrez, F. R., de Miranda Santos, I. K., Silva, J. S., et al. (2010). Tick saliva induces regulatory dendritic cells: MAPkinases and Toll-like receptor-2 expression as potential targets. Vet. Parasitol. 167, 288-297. doi: 10.1016/j.vetpar.2009.09.031

Oliveros, J. C. (2007). VENNY. An Interactive Tool for Comparing Lists With Venn Diagrams. Available online at: http://bioinfogp.cnb.csic.es/tools/venny/index. html (Accessed Aug, 2012).

Paladini, R. D., Takahashi, K., Bravo, N. S., and Coulombe, P. A. (1996). Onset of re-epithelialization after skin injury correlates with a reorganization of keratin filaments in wound edge keratinocytes: defining a potential role for keratin 16 . J. Cell Biol. 132, 381-397. doi: 10.1083/jcb.132.3.381

Patel, S., Kartasova, T., and Segre, J. A. (2003). Mouse Sprr locus: a tandem array of coordinately regulated genes. Mamm. Genome 14, 140-148. doi: 10.1007/s00335-002-2205-4

Sakuma, S., Kitamura, T., Kuroda, C., Takeda, K., Nakano, S., Hamashima, T., et al. (2012). All-trans arachidonic acid generates reactive oxygen species via xanthine dehydrogenase/xanthine oxidase interconversion in the rat liver cytosol in vitro. J. Clin. Biochem. Nutr. 51, 55-60. doi: 10.3164/jcbn.11-97

Schroeder, A., Mueller, O., Stocker, S., Salowsky, R., Leiber, M., Gassmann, M., et al. (2006). The RIN: an RNA integrity number for assigning integrity values to RNA measurements. BMC Mol. Biol. 7:3. doi: 10.1186/1471-2199-7-3

Thirumoorthy, N., Shyam Sunder, A., Manisenthil Kumar, K., Senthil Kumar, M., Ganesh, G., and Chatterjee, M. (2011). A review of metallothionein isoforms and their role in pathophysiology. World J. Surg. Oncol. 9:54. doi: 10.1186/14777819-9-54

Titus, R., Bishop, J., and Mejia, J. (2006). The immunomodulatory factors of arthropod saliva and the potential for these factors to serve as vaccine targets to prevent pathogen transmission. Parasite Immunol. 28, 131-141. doi: 10.1111/j.1365-3024.2006.00807.x

Ugajin, T., Satoh, T., Kanamori, T., Aritake, K., Urade, Y., and Yokozeki, H. (2011). FcepsilonRI, but not FcgammaR, signals induce prostaglandin 
D2 and E2 production from basophils. Am. J. Pathol. 179, 775-782. doi: 10.1016/j.ajpath.2011.04.023

Warnes, G. R., Bolker, B., Bonebakker, L., Gentleman, R., Huber, W., Liaw, A., et al. (2013). GPLOTS: Various R Programming Tools for Plotting Data. Aviiable online at: http://CRAN.R-project.org/package $=$ gplots. $\mathrm{R}$ package version 2.12.11.

Wikel, S. (2013). Ticks and tick-borne pathogens at the cutaneous interface: host defenses, tick countermeasures, and a suitable environment for pathogen establishment. Front. Microbiol. 4:337. doi: 10.3389/fmicb.2013. 00337

Wong, P., and Coulombe, P. A. (2003). Loss of keratin 6 (K6) proteins reveals a function for intermediate filaments during wound repair. J. Cell Biol. 163, 327-337. doi: 10.1083/jcb.200305032

Yamamoto, K., Kawakubo, T., Yasukochi, A., and Tsukuba, T. (2012). Emerging roles of cathepsin E in host defense mechanisms. Biochim. Biophys. Acta 1824, 105-112. doi: 10.1016/j.bbapap.2011.05.022

Zou, T., Garifulin, O., Berland, R., and Boyartchuk, V. L. (2011). Listeria monocytogenes infection induces prosurvival metabolic signaling in macrophages. Infect. Immun. 79, 1526-1535. doi: 10.1128/IAI.01195-10
Conflict of Interest Statement: The authors declare that the research was conducted in the absence of any commercial or financial relationships that could be construed as a potential conflict of interest.

Received: 13 February 2014; paper pending published: 18 March 2014; accepted: 14 April 2014; published online: 07 May 2014.

Citation: Heinze DM, Carmical JR, Aronson JF, Alarcon-Chaidez F, Wikel S and Thangamani S (2014) Murine cutaneous responses to the rocky mountain spotted fever vector, Dermacentor andersoni, feeding. Front. Microbiol. 5:198. doi: 10.3389/fmicb. 2014.00198

This article was submitted to Microbial Immunology, a section of the journal Frontiers in Microbiology.

Copyright (c) 2014 Heinze, Carmical, Aronson, Alarcon-Chaidez, Wikel and Thangamani. This is an open-access article distributed under the terms of the Creative Commons Attribution License (CC BY). The use, distribution or reproduction in other forums is permitted, provided the original author(s) or licensor are credited and that the original publication in this journal is cited, in accordance with accepted academic practice. No use, distribution or reproduction is permitted which does not comply with these terms. 